\title{
(1)
}

\section{Smoke-free legislation and acute MI}

Smoking bans improve quality of life and have substantial benefits to public health. The investigators of a study on the effect of smoke-free laws in Flanders, Belgium on deaths from acute myocardial infarction (MI) highlight interesting sex-specific differences in mortality immediately after introduction of the ban.

Unlike many other studies involving hospital data, the investigators used a population-based registry. "Populationbased registries have greater coverage, and capture a large proportion of fatal events that occur before reaching hospital," explain Tim Nawrot and Bianca Cox from Hasselt University in Belgium.

In 2006, the Belgian government introduced a smoking ban in public spaces and workplaces. This ban correlated with an almost immediate reduction in mortality attributable to acute MI, particularly in individuals aged $<60$ years. Interestingly, the reduction was more pronounced in women $(-33.8 \%$; $95 \%$ CI -49.6 to $-13.0, P<0.05)$ than men $(-13.1 \%$; $95 \% \mathrm{CI}-24.3$ to $-0.30, P<0.05)$. The smoking ban was extended to restaurants and bars in January 2007. After this second ban, mortality owing to acute MI dropped in men aged $\geq 60$ years $(-3.8 \%$; $95 \% \mathrm{CI}$ -6.5 to $-1.0, P<0.05)$.

"Banning smoking in workplaces mainly affects the working population," says Nawrot, who also notes that tobacco smoke can counteract the cardioprotective activity of oestrogen, which might explain the greater reduction in mortality among women aged $<60$ years compared with men. "Our study indicates that 1,715 deaths due to acute MI were likely prevented in Flanders between January 2006 and December 2009," highlights Nawrot. "Only $16 \%$ of the world's population is covered by smoke-free laws; [our data] further supports the introduction of global smoke-free legislation."

Tim Geach

Original article Cox, B. et al. Impact of stepwise introduction of smoke-free legislation on population rates of acute myocardial infarction deaths in Flanders, Belgium. Heart doi:10.1136/heartjnl-2014-305613 